\title{
The weber problem with regional demand ${ }^{1}$
}

\author{
E. Carrizosa ${ }^{a}$, M. Muñoz-Márquez ${ }^{b, *}$, J. Puerto $^{a}$ \\ ${ }^{a}$ Departamento de Estadistica e Investigación Operativa, Facultad de Matemáticas, c/Tarfia s/n, 41012 Sevilla, Spain \\ ${ }^{\mathrm{b}}$ Departamento de Matemáticas, Escuela Politécnica de Cádiz, c/Sacramento 82, 11002 Cádiz, Spain
}

Received 16 September 1995; accepted 27 December 1996

\begin{abstract}
This paper is devoted to the study of the Regional Weber Problem, an extension of the Weber problem which allows the demand not be concentrated onto a finite set of points.

The most serious drawback of this formulation, from a resolution viewpoint, is the high computational cost involved in the evaluation of the objective function. A new approach is proposed, which requires a low amount of computation and where it is possible to control the error on the approximation.

This approximation suggests a new methodology to solve the problem. This methodology is compared with the existing ones, showing its relevance from a practical point of view. (c) 1998 Elsevier Science B.V.
\end{abstract}

Keywords: Location; Weber problems; Average distances

\section{Introduction}

In continuous single-facility location problems one looks for the location of a facility or service optimizing some performance measure which depends on the location of the server, see e.g. [1,2].

In this paper, we address a generalization of the classical Weber problem [3] in which one seeks a location that minimizes the expected distance to a set $A$, of demand points, supposed to be distribute $\mathbb{R}^{n}$ following a probability measure $p$.

Although most existing papers suppose that the demand points constitute a discrete set, there exist

\footnotetext{
*Corresponding author. E-mail: manuel.munoz@uca.es.

${ }^{1}$ The research of the authors is partially supported by Spanish DGICYT grant PB93-0927.
}

some cases in which it is better to assume that the facilities have positive area, see [4-6] for further details.

This approach appears in a natural way in different situations. First of all, suppose that there exists uncertainty on the location of the demand; hence the demand is a random vector over a region [7-10]. Secondly, the facilities may be regions instead of points, as suggested for instance in [11]. Finally, regional models fit better when there exists a very large number of demand points, making the discrete problem cumbersome [12].

In order to apply these models an important drawback needs to be avoided, the efficient evaluation of the expected distances. In some cases, one can evaluate the expected distance to the region involved, (see [13-16] for rectangular regions or [17] 
for polygonal regions) or one can evaluate the gradient of the expected distance, see [4]. When this does not happen, the usual approach has consisted of replacing such distances by an approximation with a simpler form. However, this methodology presents the serious pitfall that the optimal solutions of the approximate problem are hard to relate with the optimal solution of the original problem. All of this leads us to propose a new methodology which keeps the error under control and requires a moderate computational effort.

The rest of this paper is organized as follows. The Section 2 is devoted to the development of some theoretical properties of the problem under study, reducing the problem to a convex program. Section 3 analyzes the existing methodologies for this problem, showing their drawbacks, and a new approach is proposed and compared with previous attempts. Finally, Section 4 concludes the paper.

\section{Formulation and theoretical properties}

The location model we are interested in is formulated as

$\min _{x \in \mathbb{R}^{n}} \int \gamma(x-a) \mathrm{d} p(a)$

where $x$ is the location of the facility, $y$ a gauge, see $[18,19]$, i.e., there exists a non-empty, convex, compact set $C$, the interior of which contains the origin, such that

$\gamma(x):=\inf \{\lambda \geqslant 0: x \in \lambda C\}$,

and $p$ is a probability measure over a bounded set $A \subset \mathbb{R}^{n}$.

It should be noted that if $p$ is a discrete measure the problem reduces to the classical Weber problem.

Once the model has been formulated the rest of this section is devoted to the study of theoretical properties of the objective function, which allow the resolution of the problem using standard algorithms for convex optimization.

Let $\overline{\mathrm{d}}(\cdot, A): \mathbb{R}^{n} \rightarrow[0,+\infty]$ be the function defined by $\overline{\mathrm{d}}(x, A)=\int \gamma(x-a) \mathrm{d} p(a)$.

Then the following property on $\overline{\mathrm{d}}$ is well known, see $[4,20,17]$.

Property 2.1. The function $\overline{\mathrm{d}}(\cdot, A)$ is finite, convex and hence continuous.

The following result states some useful properties of the level sets of the objective function.

Property 2.2. The level sets of $\overline{\mathrm{d}}(\cdot, A)$ are compact, convex sets. Hence $\overline{\mathrm{d}}(\cdot, A)$ is inf-compact.

Proof. The function $\overline{\mathrm{d}}(\cdot, A)$ is continuous and convex, thus the level sets are closed and convex. It remains to show that they are also bounded.

Let $R>0$ and let $L(R)=\left\{x \in \mathbb{R}^{n}: \overline{\mathrm{d}}(x, A) \leqslant R\right\}$. If $L(R)=\emptyset$, the theorem holds. If $L(R)$ is not empty, for any point $x$ in $L(R)$, then

$$
\begin{aligned}
R \geqslant \overline{\mathrm{d}}(x, A) & =\int \gamma(x-a) \mathrm{d} p(a) \\
& \geqslant \int(\gamma(x)-\gamma(\mathrm{a})) \mathrm{d} p(\mathrm{a}) \\
& =\gamma(x)-\int \gamma(\mathrm{a}) \mathrm{d} p(a) \\
& =\gamma(x)-\overline{\mathrm{d}}(0,-A),
\end{aligned}
$$

where 0 is the origin.

Hence $\gamma(x) \leqslant R+\overline{\mathrm{d}}(0,-A)<+\infty$, thus $L(R)$ is a subset of the ball of $\gamma$ centered at the origin with radius $R+\overline{\mathrm{d}}(0,-A)$. Thus $L(R)$ is bounded.

Using this result one obtains Corollary 2.1.

Corollary 2.1. The set of optimal solution to (1) is a non-empty, compact, convex set.

These properties allows us to solve the problem by algorithms of optimization of convex functions that only need the evaluation of the objective function, such as the coordinate descent method, see [21]. But, as shown in Section 3, the high computational cost associated with the evaluation of the objective function (namely the numerical evaluation of a bidimensional integral), leads us to address the problem from a different perspective. 


\section{Approximating average distances}

As we point out in previous sections the evaluation of the expected distances becomes a crucial step in the resolution of (1). As far as we know, only two different deterministic approaches have been proposed in the literature of location: the approximation of regions by their centroids and by disks, see [22] for a review of these methodologies and see [17] for a non-deterministic approach. We devote the following subsection to describe these approaches.

\subsection{Approximation by centroids and disks}

In real problems the distribution of the demand over $A$ is not so simple, and the evaluation of the expected distance can be simplified if the original probability measure of the demand can be seen or approximated with arbitrary accuracy by a mixture of distributions. This is the case when the demand is distributed over a union of $k$ different sets $A_{i}$, being the distribution $p_{i}$ on each $A_{i}$, simple, e.g. the demand uniformly distributed on each of a set of rectangles. Thus (1) may be formulated as

$\min _{x \in \mathbb{R}^{n}} \sum_{i=1}^{k} w_{i} \int_{A_{i}} \gamma(x-a) \mathrm{d} p_{i}(a)$,

where for each $i=1, \ldots, k, p_{i}$ is a probability measure over $A_{i}$ and $w_{i}$ represents the relative weight of the probability mixture on each subregion $A_{i}$. The above problem may be formulated equivalently as

$\min _{x \in \mathbb{R}^{n}} \sum_{i=1}^{k} w_{i} \overline{\mathrm{d}}\left(x, A_{i}\right)$

The simplest way to approximate this problem is to substitute each region $A_{i}$ by its centroid $c_{i}$, and the $\overline{\mathrm{d}}\left(x, A_{i}\right)$ by $\gamma\left(x-c_{i}\right)$, leading to the Weber problem

$\min _{x \in \mathbb{R}^{n}} \sum_{i=1}^{k} w_{i} \gamma\left(x-c_{i}\right)$.

Aly [23] and Vaughan [16] show that this methodology is not satisfactory, because the practical behavior of this methodology is strongly dependent of the norm in use, (e.g. usually good for the Euclidean norm and not so good for the $l_{1}$ norm), and the shape of the demand region. In addition what is worse, is that the error of such approximation is completely unknown but not necessarily small.

In order to obtain a more precise approximation, the next step might be to replace $A_{i}$ by other simpler region, such as a disk. Among others, Drezner [12], Koshizuka and Kurita [11] and Stone [15], have proposed to replace each subregion $A_{i}$ by a disk $C_{i}$ centered at the centroid and with the same area. This leads to solve the problem

$\min _{x \in \mathbb{R}^{n}} \sum_{i=1}^{k} w_{i} \overline{\mathrm{d}}\left(x, C_{i}\right)$

Vaughan, in the above mentioned paper, studies such substitution, concluding that the approximation does not work properly for some regions.

From a practical viewpoint there exists another serious drawback: the expected distance to a disk is known only for special gauges and even in these cases, the expressions obtained are very cumbersome and must be approximated. In addition, in these methodologies, the question of how large the errors are remains still open. This question must be answered through computational studies.

\subsection{Approximating the probability measure}

The objective of this section is to find an approximation to the expected distances $\overline{\mathrm{d}}(\cdot, A)$ substituting the region $A$, such that the error is kept under control.

To do this we propose to find a region $R \subset \mathbb{R}^{n}$ verifying:

1. $\overline{\mathrm{d}}(\cdot, A) \simeq \overline{\mathrm{d}}(\cdot, R)$,

2. the evaluation of $\mathrm{d}(\cdot, R)$ is much easier than the evaluation of $\overline{\mathrm{d}}(\cdot, A)$.

Let $R$ be a set, such that $p(R)>0$. Then the expected distance from $x$ to $R$ is given by

$\overline{\mathrm{d}}(x, R)=\frac{1}{p(R)} \int_{R} \gamma(x-a) \mathrm{d} p(a)$. 
Let $\quad \delta(x)=\sup _{a \in A} \gamma(x-a), e(x, R)=\mid \overline{\mathrm{d}}(x, A)$ - $\overline{\mathrm{d}}(x, R) \mid$ be the error when $A$ is replaced by $R$, and $\hat{e}(x, R)=((1-p(R)) / p(R)) \delta(x)$. Observe that $\hat{e}$ is finite since $A$ is, by assumption, a bounded set.

The Lemma 3.1 shows that $\hat{e}$ is an upper bound of $e$.

Lemma 3.1. One has $e(x, R) \leqslant \hat{e}(x, R)$.

Proof. First,

$$
\begin{aligned}
& \overline{\mathrm{d}}(x, R)-\overline{\mathrm{d}}(x, A) \\
& =\frac{1}{p(R)} \int_{R} \gamma(x-a) \mathrm{d} p(a)-\int_{A} \gamma(x-a) \mathrm{d} p(a) \\
& =\frac{1}{p(R)}\left(\int_{R} \gamma(x-a) \mathrm{d} p(a)-p(R) \int_{A} \gamma(x-a) \mathrm{d} p(a)\right) \\
& \leqslant \frac{1}{p(R)}\left(\int_{A} \gamma(x-a) \mathrm{d} p(a)-p(R) \int_{A} \gamma(x-a) \mathrm{d} p(a)\right) \\
& =\frac{1-p(R)}{p(R)} \int_{A} \gamma(x-a) \mathrm{d} p(a) \\
& \leqslant \frac{1-p(R)}{p(R)} \sup _{a \in A} \gamma(x-a) .
\end{aligned}
$$

On the other hand

$$
\begin{aligned}
& \overline{\mathrm{d}}(x, A)-\overline{\mathrm{d}}(x, R) \\
& =\int_{A} \gamma(x-a) \mathrm{d} p(a)-\frac{1}{p(R)} \int_{R} \gamma(x-a) \mathrm{d} p(a) \\
& =\frac{1}{p(R)}\left(p(R) \int_{A} \gamma(x-a) \mathrm{d} p(a)-\int_{R} \gamma(x-a) \mathrm{d} p(a)\right) \\
& \leqslant \frac{1}{p(R)}\left(\int_{A} \gamma(x-a) \mathrm{d} p(a)-\int_{R} \gamma(x-a) \mathrm{d} p(a)\right) \\
& =\frac{1}{p(R)} \int_{A \backslash R} \gamma(x-a) \mathrm{d} p(a)
\end{aligned}
$$

$$
\begin{aligned}
& \leqslant \frac{p(A \backslash R)}{p(R)} \sup _{a \in A \backslash R} \gamma(x-a) \\
& \leqslant \frac{1-p(R)}{p(R)} \sup _{a \in A} \gamma(x-a) .
\end{aligned}
$$

Hence

$$
e(x, R) \leqslant \frac{1-p(R)}{p(R)} \sup _{a \in A} \gamma(x-a) .
$$

If one may control the error of the approximation, then the problem would be solvable with any given accuracy. Therefore, the next step is the development of conditions which assure that the error converges to zero. Obviously, the convergence of the error to zero can be obtained by forcing its upper bound to converge to zero. Observe that $\hat{e}(x, R)$ is non-increasing in $p(R)$ and converging to zero when $p(R)$ converges to 1 . So, the error can be taken as small as desired.

Theorem 3.1. Let $\epsilon, \delta>0$. If $R$ is such that $p(R) \geqslant \delta /(\epsilon+\delta)$ then $\sup \{e(x, R): \delta(x) \leqslant \delta\} \leqslant \epsilon$.

Proof. Consider $x$ such that $\delta(x) \leqslant \delta$. By Lemma 3.1 one has that

$e(x, R) \leqslant \hat{e}(x, R)=\frac{1-p(R)}{p(R)} \delta(x) \leqslant \frac{1-p(R)}{p(R)} \delta$.

By assumption $p(R) \geqslant \delta /(\epsilon+\delta)$ which implies $((1-p(R)) / p(R)) \delta \leqslant \epsilon$ and the result follows.

Remark that this result gives a uniform bound for the error $e$.

\subsection{An example}

In this example we use the above results in a problem in which $\gamma$ is the Euclidean norm and the demand is distributed over a union of elliptical regions in the plane, showing first how to approximate the expected distance to an ellipse and then comparing such approximation with previous approaches. 


\subsubsection{Expected distances to an ellipse}

We assume that, given $x \in \mathbb{R}^{2}$ and a triangle $T$, we know how to evaluate exactly the expected distance $\overline{\mathrm{d}}(x, T)$, see [17] for an algorithm.

The problem addressed in this section is the evaluation of the expected distances from a point $x \in \mathbb{R}^{2}$ to another point uniformly distributed in an ellipse centered at the origin with $r_{x}$ and $r_{y}$ as half of the length of the axes. This average distance can be approximated by replacing each ellipse by a polygon with $m$ sides.

To do this, let $P_{l}=\left(r_{x} \cos (2 \pi l / m), r_{y} \sin (2 \pi l)\right.$ $m)$ ) for each $l=0, \ldots, m$ and $T_{l}$ be the triangle with vertices $0, P_{l}$ and $P_{l+1}$ for $l=0, \ldots, m-1$. Then $R_{m}:=\bigcup_{l=0}^{m-1} T_{l}$ is a polygon with $m$ sides.

Since the area of these triangles are $r_{x} r_{y} / 2 \sin (2 \pi / m)$ then the area of $R_{m}$ is $m r_{x} r_{y} / 2$ $\sin (2 \pi / m)$, and then $p\left(R_{m}\right)=m /(2 \pi) \sin (2 \pi / m)$. By Lemma 3.1, one obtains

$\hat{e}\left(x, R_{m}\right)=\left(\frac{2 \pi}{m \sin \left(\frac{2 \pi}{m}\right)}-1\right) \delta(x)$.

Remark 3.1. The error depends on the number of sides and the gauge used only through the highest distance from $x$ to the ellipses.

Although for this case one could improve the bound of the error by exploiting the geometry of the problem, it should be noted that this bound is quite good. Indeed, it is easily checked that, taking limit in $m$,

$\lim _{m \rightarrow+\infty} m^{2} \hat{e}\left(x, R_{m}\right)=\frac{2}{3} \pi^{2} \delta(x)$,

thus $\hat{e}\left(x, R_{m}\right)$ is of the same order than $1 / \mathrm{m}^{2}$.

Theorem 3.1 implies that the error can be chosen as small as desired taking a sufficiently large number of triangles. However, a large number of sides involves a high computational cost. The rest of this section is devoted to illustrate the fact that the results are very good even when the number of sides considered is not too large.

If the scalar $\delta(x)$ is fixed for any given problem, then the error behaves like

$\hat{\epsilon}\left(R_{m}\right)=\frac{2 \pi}{m \sin \left(\frac{2 \pi}{m}\right)}-1$.

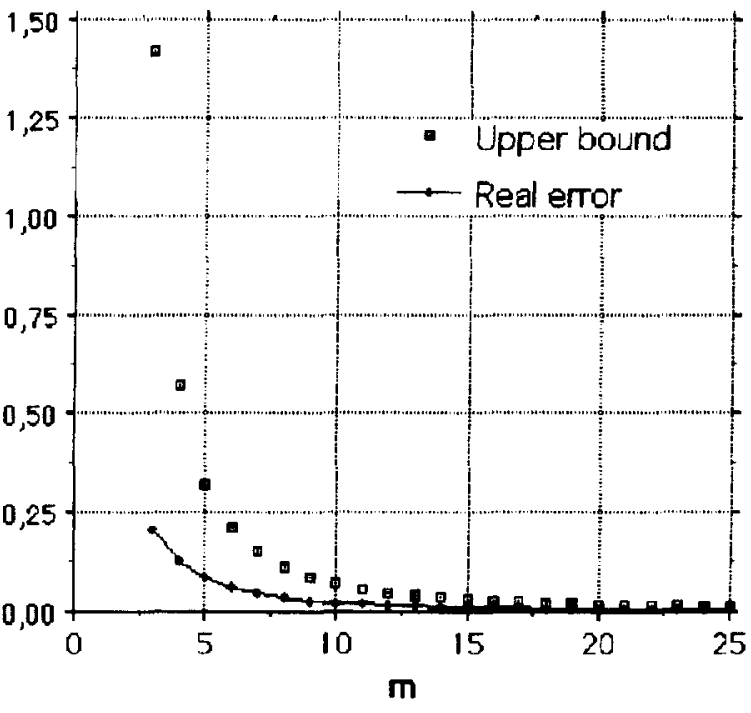

Fig. 1. Real error.

A simple computational study allows us to figure out that the real errors are very small with respect to the upper bounds which are themselves also small, $\left(\hat{\epsilon}\left(R_{10}\right) \simeq 0.07\right.$ and $\left.\hat{\epsilon}\left(R_{25}\right) \simeq 0.01\right)$. This fact is shown in Figure 1, where we represent such values for the expected distances from the origin to the unit disk centered at the origin.

The percentage of error is estimated using the value obtained replacing the disks with a polygon with 1000 sides. This is admissible approximation since the error is lower than $\hat{\epsilon}\left(R_{1000}\right)=$ 0.00000658 . Thus, the real errors $e\left(R_{10}\right)=0.02164$ and $e\left(R_{25}\right)=0.003499$, represent relative errors of $3.25 \%$ and $0.52 \%$, respectively, whereas the upper bound suggests relative errors of $10.34 \%$ and $1.59 \%$. Different examples show similar ratios between the error and its upper bound.

Table 1 shows the computational time (in milliseconds) needed to get the error in column "Error" using the number of triangles in colums "sides". Hence, the low computational cost allows us to recommend the use of triangular approximations instead of other procedures.

\subsubsection{Resolution and comparison}

Koshizuka and Kurita [11] have proposed to solve the problem of piecewise uniform regional demand by replacing each region by a disk. At 
Table 1

Triangular approximation

\begin{tabular}{lrr}
\hline Error & Sides & ms \\
\hline 1 & 4 & 0 \\
0.1 & 9 & 0 \\
0.01 & 26 & 11 \\
0.001 & 82 & 22 \\
0.0001 & 257 & 99 \\
0.00001 & 812 & 275 \\
\hline
\end{tabular}

the same time they propose an approximation of the expected distance to a point uniformly distributed on a disk using Taylor expansions.

This section is devoted to compare the disk approximation and our methodology. In order to do that, we consider the location problems in [16] (and some of them slightly modified) to evaluate the approximation of expected distances to regions by the expected distances to disk.

The problems are depicted in Fig. 2. The demand is a mixture of uniform distributions over rectangles, such that the weight of each rectangle is equal to its area. The solutions are given in Table 2, where:

- $(x, y)$ is the exact solution of the problem.

- $z$ is the objective function at point $(x, y)$.

- $\left(x_{c}, y_{c}\right)$ is the solution of the problem replacing each rectangle by a disk.

- $z_{c}$ is the (exact) objective function at point $\left(x_{c}, y_{c}\right)$.

- $e_{c}$ is the percentage of error, $100\left(z_{c}-z\right) / z$.

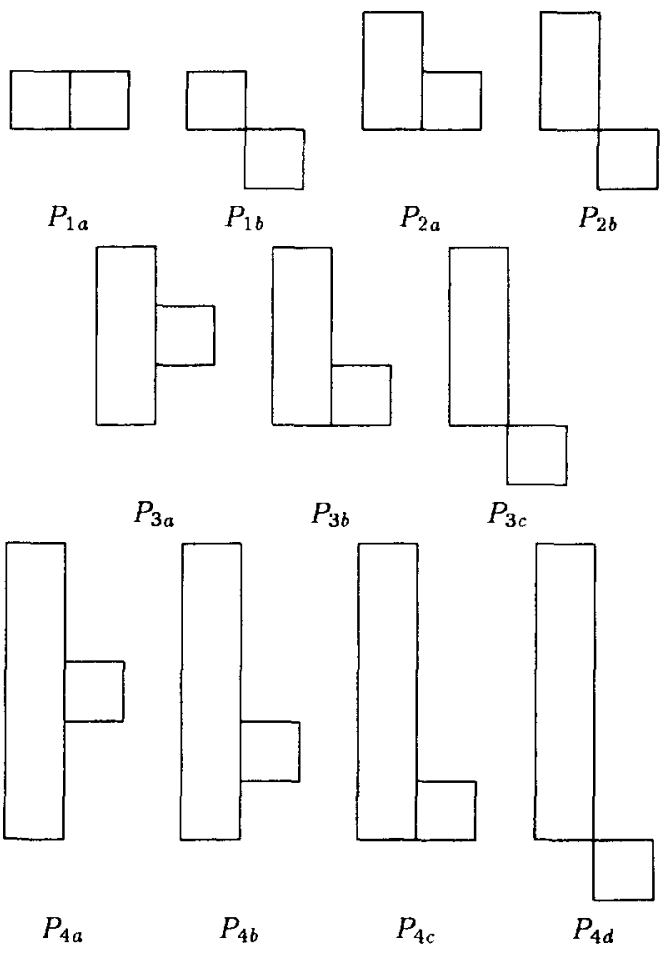

Fig. 2. Example problems.

- $\left(x_{3}, y_{3}\right)$ is the solution of the problem replacing each rectangle by a disk, and then replacing each disk by a triangle.

- $z_{3}$ is the (exact) objective function at point $\left(x_{3}, y_{3}\right)$.

- $e_{3}$ is the percentage of error, $100\left(z_{3}-z\right) / z$.

Table 2

Solutions of example problems

\begin{tabular}{llllllllllll}
\hline Problem & $P_{1 a}$ & $P_{1 b}$ & $P_{2 a}$ & $P_{2 b}$ & $P_{3 a}$ & $P_{3 b}$ & $P_{3 c}$ & $P_{5 a}$ & $P_{5 b}$ & $P_{5 c}$ & $P_{5 d}$ \\
\hline$x$ & 1.000 & 1.000 & 0.806 & 0.500 & 0.756 & 0.706 & 0.635 & 0.703 & 0.663 & 0.607 & 0.574 \\
$y$ & 0.500 & 0.000 & 0.806 & 0.500 & 1.500 & 1.179 & 1.059 & 2.500 & 2.191 & 2.067 & 2.030 \\
$z$ & 1.187 & 1.530 & 2.169 & 2.469 & 3.391 & 3.731 & 4.500 & 7.459 & 7.800 & 8.551 & 9.453 \\
& & & & & & & & & & & 1.234 \\
$x_{3}$ & 0.978 & 1.042 & 1.095 & 0.482 & 1.154 & 1.167 & 1.180 & 1.219 & 1.213 & 1.219 \\
$y_{3}$ & 0.500 & -0.028 & 0.732 & 0.120 & 1.500 & 0.861 & 0.133 & 2.500 & 1.822 & 1.022 & 0.327 \\
$z_{3}$ & 1.187 & 1.531 & 2.286 & 2.612 & 3.668 & 4.159 & 5.631 & 8.053 & 8.563 & 10.372 & 12.946 \\
$e_{3}(\%)$ & 0.04 & 0.06 & 5.37 & 5.77 & 8.18 & 11.42 & 25.16 & 7.96 & 9.91 & 21.30 & 36.95 \\
$x_{c}$ & 1.000 & 1.000 & 1.086 & 0.500 & 1.134 & 1.134 & 1.134 & 1.191 & 1.191 & 1.191 & 1.191 \\
$y_{c}$ & 0.500 & 0.000 & 0.707 & 0.121 & 1.500 & 0.866 & 0.233 & 2.500 & 1.810 & 1.117 & 0.428 \\
$z_{c}$ & 1.187 & 1.530 & 2.283 & 2.611 & 3.643 & 4.111 & 5.441 & 7.992 & 8.526 & 10.103 & 12.594 \\
$e_{c}(\%)$ & 0.00 & 0.00 & 5.22 & 5.72 & 7.41 & 10.19 & 20.93 & 7.15 & 9.44 & 18.15 & 33.23 \\
\hline
\end{tabular}




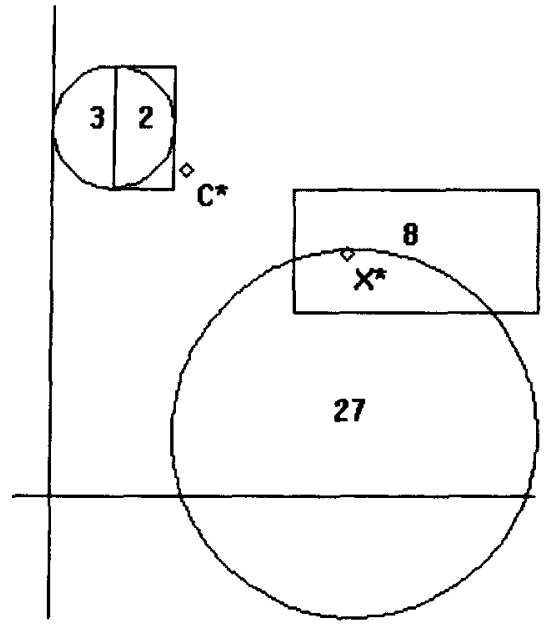

Fig. 3. Disks and rectangles example.

Note that when we replace the disks involved by triangles, the solution of this problem is very close to the disk problem solution. This confirms the fact that a disk can be replaced by a regular polygon, even when it has a small number of sides.

In order to complete these examples, we want to include one more that involves not only rectangles: Consider that we have four types of demands, each of that is uniformly distributed over regions $R_{1}, R_{2}, R_{3}$ and $R_{4}$, respectively, with weights of $8,2,3$ and 27. See the problem depicted in Fig. 3 (the number inside each figure is its weight).

The point $C^{*}=(2.2148,5.2993)$ is the solution of the disk approximation problem, and $X^{*}=$ $(4.8823,3.9729)$ is the solution of the original problem. The expected mean distance from $X^{*}$ is 43.1867 and the expected distance from $C^{*}$ is 55.2264 . This is $27.88 \%$ higher.

Note that, even in these simple examples, we have found cases in which the percentages of errors are very high when we replace regions by disks.

Regarding the computational cost, we can say that the exact problem has been solved in $0.1 \mathrm{~s}$, in the worst case, using the easily implementable coordinatewise descent method, see [21].

From this, one should expect that very large real problems can be solved in a moderate amount of time, by replacing regions by polygons, as done by Geographical Information Systems (GIS).

\section{Conclusions}

This paper is devoted to the study of the Weber problem with regional demand, which is an extension of the standard Weber problem in which the demand is not assumed to be concentrated at a finite set, but follows an arbitrary probability measure.

We develop some properties of the objective function that allow us to solve the problem by existing techniques of convex optimization, but, as it is remarked, the evaluation of the objective function has a high computational cost. This problem is solved through a new approximation of the objective function, consisting of replacing the demand distribution by a simpler one. This methodology enables us to keep the error under control.

Some examples show that the requirements are very moderate. In fact with a small computational effort one can obtain good results. This fact suggests that this methodology may be applied to real problems.

\section{References}

[1] R.L. Francis, L.F. McGinnis, J.A. White, Facility Layout and Location: An Analytical Approach, Prentice Hall, Englewood Cliffs, NJ, 1992.

[2] F. Plastria, Continuous location anno 1992, a progress report, Studies in Locationa Analysis, 5 (1993) 85-128.

[3] R.F. Love, J.G. Morris, G.O. Wesolowsky, Facilities Location: Models and Methods, North-Holland, Amsterdam, 1988.

[4] E. Carrizosa, E. Conde, M. Muñoz-Márquez, J. Puerto, The generalized Weber problem with expected distances, RAIRO Recherche Opérationnelle Operations Research, 29 (1995) 35-57.

[5] G.O. Weslowsky, R.F. Love, Location of facilities with rectangular distances among point and area destinations, Naval Research Logistics Quarterly, 18 (1971) 83-90.

[6] G.O. Weslowsky, R.F. Love, A nonlinear approximation method for solving a generalized rectangular distance Weber problem, Management Science, 18 (11) (1972) 656-663.

[7] Z. Drezner, G.O. Wesolowsky, Optimal location of a facility relative to area demands, Naval Research Logistics Quarterly, 27 (2) (1980) 199-206.

[8] Z. Drezner, Sensitivity analysis of the optimal location of a facility, Naval Research Logistics Quarterly, 32 (1985) 209-224.

[9] Z. Drezner, G.O. Wesolowsky, Optimum location probabilities in the $l_{p}$ distance Weber problem, Transportation Science, 15 (1981) 923-931. 
[10] H. Juel, Bounds in the generalized Weber problem under conditions of uncertainty, Operational Research, 29 (1981) 1219-1227.

[11] T. Koshizuka, O. Kurita, Approximate formulas of average distances associated with regions and their applications to location problems, Mathematical Programming, 52 (1991) 99-123.

[12] Z. Drezner, Location of regional facilities, Naval Research Logistics Quarterly, 33 (1986) 523-529.

[13] A.A. Aly, A.S. Marucheck, Generalized Weber problem with rectangular regions, Journal of Operational Research Society, 33 (1982) 983-989.

[14] R.F. Love, A computational prcedure for optimally locating a facility with respect to several rectangular regions, Journal of Regional Science, 12 (1972) 233-242.

[15] R.E. Stone, Some Average distance results, Transportation Science, 25 (1) (1991) 83-91.

[16] R. Vaughan, Approximate formulas for average distances associated with zones, Transportation Science, 18 (3) (1984) 231-244.
[17] M. Muñoz-Márquez, El Problema de Weber Regional, Ph.D. Thesis, Universidad de Sevilla, 1995.

[18] C. Michelot, The mathematics of continuous location, Studies in Locationa Analysis, 5 (1993) 59-84.

[19] R.T. Rockafellar, Convex Analysis, Princeton University Press, Princeton, NJ, 1970.

[20] C.J. Witzgall, Optima location of a central facility, mathematical models and concepts, Technical Report 8388, National Bureau of Standards, 1964.

[21] D.E. Luenberger, Linear and Nonlinear Programming, 2nd ed., Addison-Wesley, Reading, MA, 1989.

[22] J. Puerto-Albandoz, M. Muño-Márquez, Lecturas En Teoría de Localización, chapter problema de Localización Estocástica Continua, Secretariado de Publicaciones de la Universidad de Sevilla, 1996, pp. 167-195.

[23] A.A. Aly, Probabilistic Formulations of some Facility Location Problems, Ph.D. Thesis, Virginia Polytechnic Institute and State University, 1974. 